\title{
Dielectric assist accelerating structure
}

\author{
D. Satoh, ${ }^{1, *}$ M. Yoshida, ${ }^{2}$ and N. Hayashizaki ${ }^{3}$ \\ ${ }^{1}$ Graduate School of Science and Engineering, Tokyo Institute of Technology, \\ Meguro, Tokyo 152-8550, Japan \\ ${ }^{2}$ High Energy Accelerator Research Organization KEK, 1-1 Oho, Tsukuba, Ibaraki 305-0801, Japan \\ ${ }^{3}$ Research Laboratory for Nuclear Reactors, Tokyo Institute of Technology, \\ Meguro, Tokyo 152-8550, Japan
}

(Received 7 October 2015; published 25 January 2016)

\begin{abstract}
A higher-order $\mathrm{TM}_{02 n}$ mode accelerating structure is proposed based on a novel concept of dielectric loaded rf cavities. This accelerating structure consists of ultralow-loss dielectric cylinders and disks with irises which are periodically arranged in a metallic enclosure. Unlike conventional dielectric loaded accelerating structures, most of the rf power is stored in the vacuum space near the beam axis, leading to a significant reduction of the wall loss, much lower than that of conventional normal-conducting linac structures. This allows us to realize an extremely high quality factor and a very high shunt impedance at room temperature. A simulation of a 5 cell prototype design with an existing alumina ceramic indicates an unloaded quality factor of the accelerating mode over 120000 and a shunt impedance exceeding $650 \mathrm{M} \Omega / \mathrm{m}$ at room temperature.
\end{abstract}

DOI: 10.1103/PhysRevAccelBeams.19.011302

\section{INTRODUCTION}

Over many years, conventional disk-loaded copper structures have been a subject of much research and implementation, whose extreme cases of performance have been reported, for instance, in $[1,2]$ which have demonstrated a high accelerating gradient of up to $100 \mathrm{MV} / \mathrm{m}$ at the X-band frequency. However, compared to superconducting cavities which realize a much higher quality factor $\left(Q_{0}\right)$ and a higher shunt impedance $\left(R_{\mathrm{sh}}\right)$, the room temperature rf structures are substantially less advantageous in terms of the power efficiency. A room-temperature rf linac structure with $Q_{0}$ and $R_{\mathrm{sh}}$ much higher than those of present conventional normal-conducting rf linac structures, if realized, would put forward a major breakthrough for future accelerators in material and radiation processing $[3,4]$, cancer therapy [5] or imaging [6], as well as the basic research such as high energy electron-positron colliders. Such a high-performance linac structure, however, has not been established yet, and therefore there is still a large amount of room for the technological innovation of accelerating structures.

A potential alternative to conventional disk-loaded copper structures is dielectric loaded accelerating (DLA) structures [7-9]. A DLA structure comprises a simple geometry where a dielectric tube is surrounded by a conducting cylinder, and it works as a slow wave

\footnotetext{
*satou.d.ad@m.titech.ac.jp

Published by the American Physical Society under the terms of the Creative Commons Attribution 3.0 License. Further distribution of this work must maintain attribution to the author $(s)$ and the published article's title, journal citation, and DOI.
}

structure for beam acceleration whose phase velocity of the traveling wave is equal to or less than the speed of light in free space. Since these basic theory of $\mathrm{rf}$ driven DLA structures were first proposed in the 1940's [10-12], numerous studies have examined the use of dielectric materials in accelerating structures [13-16]. Recently, new concepts of dielectric based accelerating structures which improved the accelerator performance such as a dual-layered DLA structure [15], a hybrid dielectric and iris loaded periodic accelerating structure [16] and a multilayered DLA structure $[17,18]$ which is operated by a higher order mode were proposed, and the possibilities of DLA structures are gradually expanding. In addition to the background of improving the accelerator performance of DLA structures, they are presently drawing renewed attention due to remarkable progress in new ceramic materials with high dielectric permittivity $(\varepsilon>20)$ and low loss $(Q \sim 40,000)$ [19-21], and, more recently, in ultralow-loss types [22-24].

The simplicity of the DLA structure offers a great advantage for high frequency ( $>\mathrm{X}$-band) accelerating structures as compared with conventional metal structures which demand extremely tight fabrication tolerances [7]. Moreover, there are other advantages of ease of damping the beam-induced deflection modes which can lead to a beam instability in DLA structures effectively by simple structure [25]. However, the accelerating mode in a DLA structure, whose phase velocity has to be matched to the particle speed, is limited to evanescent modes. Therefore, in a DLA structure, most of the rf energy is stored in a dielectric region and its energy cannot be effectively utilized for beam acceleration. Thus, it is difficult to much improve the shunt impedance of its 
DLA structures, and there is still room for improvement on these dielectric based accelerating structures.

This paper presents the concept of a novel dielectric loaded rf accelerating structure, which arranges a dielectric structure operating in the $\mathrm{TM}_{02 n}$ mode within a metallic enclosure. We call this structure the "Dielectric Assist Accelerating (DAA) Structure," since the accelerator performance is drastically improved by a novel dielectric structure. In this new cavity structure, the electromagnetic field distribution of accelerating mode $\left(\mathrm{TM}_{02 n}\right.$ mode) can be controlled to greatly reduce the wall loss on metallic surfaces as compared to that of a $\mathrm{TM}_{01}$ mode pillbox cavity. Thus, a high quality factor and a high shunt impedance are simultaneously realized. In Sec. II, we present the principle of the DAA structure and describe its features. In Secs. III and IV, we show the design of regular cells and end cells of a DAA structure. Section $V$ presents a practical design of a prototype 5 cell DAA structure and which may be built for demonstration purposes.

\section{CONCEPT OF DAA STRUCTURE}

Conceptual illustrations of a DAA structure are shown in Fig. 1. The DAA structure is a $\mathrm{TM}_{02 n}$ mode standing wave
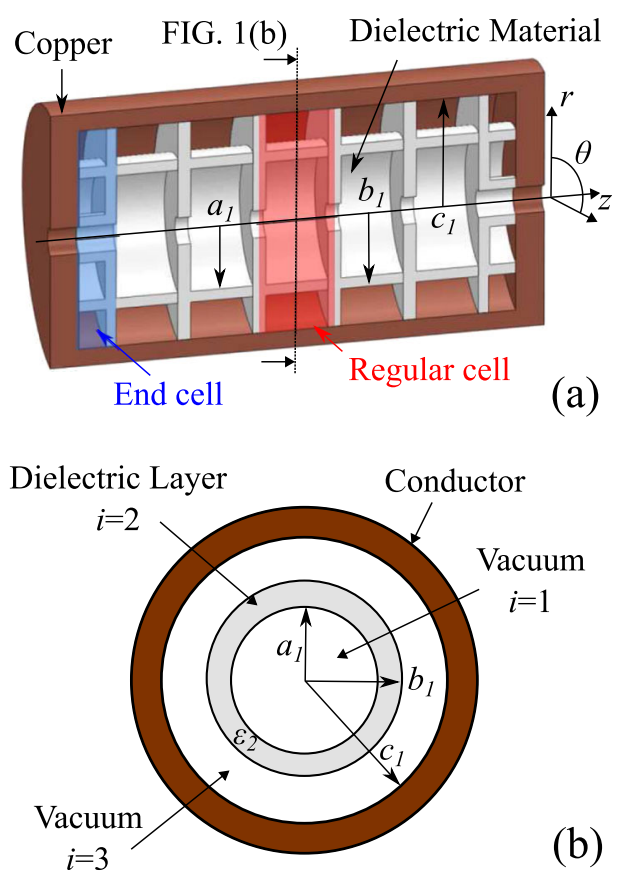

FIG. 1. Conceptual illustrations of a DAA structure. (a) Longitudinal cross section of the 5 cell prototype design of the DAA structure. (b) Cross section of the DAA structure perpendicular to the beam direction and parallel to dielectric disks at the center of the regular cell. The symbol " $i$ " denotes the index of regions (vacuum or dielectric regions) running from 1 (center) through 3 (outermost). accelerating structure which consists of ultralow-loss dielectric cylinders and disks with iris periodically arranged in a metallic enclosure. This structure comprises two types of cell structures: a "regular cell" and an "end cell." The regular cells provide the beam with accelerating field. While the end cell on each end of the structure serves to reduce the rf dissipation on end plates and they do not contribute to beam acceleration.

Figure 1(b) shows the cross section of the DAA structure perpendicular to the beam direction and parallel to dielectric disks at the center of regular cell. It is a cylindrical geometry partially occupied by a dielectric material with an inner radius $a_{1}$, an outer radius $b_{1}$ which is placed inside a cylindrical metallic waveguide of a radius $c_{1}$.

Now, we consider only the propagation mode in both the vacuum regions $(i=1,3)$ and the dielectric layer $(i=2)$ of this waveguide which does not contain dielectric disks. The general solutions for the electromagnetic field of $\mathrm{TM}_{0 m}$ modes propagating to along the $+z$ direction through the $i$ th layer can be expressed by

$$
\begin{aligned}
E_{z}^{i}(r, z, t) & =\left\{A_{i} J_{0}\left(k_{i} r\right)+B_{i} Y_{0}\left(k_{i} r\right)\right\} e^{j\left(\omega t-k_{z} z\right)} \\
E_{r}^{i}(r, z, t) & =\frac{j k_{z}}{k_{i}}\left\{A_{i} J_{1}\left(k_{i} r\right)+B_{i} Y_{1}\left(k_{i} r\right)\right\} e^{j\left(\omega t-k_{z} z\right)} \\
H_{\theta}^{i}(r, z, t) & =\frac{j \omega \varepsilon_{i}}{k_{i}}\left\{A_{i} J_{1}\left(k_{i} r\right)+B_{i} Y_{1}\left(k_{i} r\right)\right\} e^{j\left(\omega t-k_{z} z\right)},
\end{aligned}
$$

where $A_{i}$ and $B_{i}$ are the field amplitudes in the $i$ th layer. The cutoff wave number $k_{i}$ is given by

$$
k_{i}=\sqrt{\omega^{2} \varepsilon_{i} \mu_{i}-k_{z}^{2}},
$$

where $k_{z}$ is the propagation coefficient along the $z$ axis, $\varepsilon_{i}$ and $\mu_{i}$ are permittivity and permeability in the $i$ th layer, respectively. $J_{l}$ and $Y_{l}$ are the $l$ th-order Bessel functions of the first and second kind, respectively. By applying the boundary conditions at each of the radial boundaries, the following equations are obtained:

$$
\left\{\begin{array}{l}
S\left(b_{1}\right) R\left(a_{1}\right) M_{4} M_{3} M_{2}\left(A_{1} \vec{V}_{1}\right)=\left(\begin{array}{l}
A_{3} \\
B_{3}
\end{array}\right) \\
A_{3} J_{0}\left(k_{3} c_{1}\right)+B_{3} Y_{0}\left(k_{3} c_{1}\right)=0 .
\end{array}\right.
$$

The functions, matrices and a vector in the Eq. (2) are expressed by 


$$
\begin{aligned}
R\left(a_{1}\right) & =\frac{1}{J_{0}\left(k_{2} a_{1}\right) Y_{1}\left(k_{2} a_{1}\right)-J_{1}\left(k_{2} a_{1}\right) Y_{0}\left(k_{2} a_{1}\right)} \\
S\left(b_{1}\right) & =\frac{1}{J_{0}\left(k_{3} b_{1}\right) Y_{1}\left(k_{3} b_{1}\right)-J_{1}\left(k_{3} b_{1}\right) Y_{0}\left(k_{3} b_{1}\right)} \\
\vec{V}_{1} & =\left(\begin{array}{c}
J_{0}\left(k_{1} a_{1}\right) \\
\frac{\varepsilon_{1}}{k_{1}} J_{1}\left(k_{1} a_{1}\right)
\end{array}\right) \\
M_{2} & =\left(\begin{array}{cc}
Y_{1}\left(k_{2} a_{1}\right) & -\frac{k_{2}}{\varepsilon_{2}} Y_{0}\left(k_{2} a_{1}\right) \\
-J_{1}\left(k_{2} a_{1}\right) & \frac{k_{2}}{\varepsilon_{2}} J_{0}\left(k_{2} a_{1}\right)
\end{array}\right) \\
M_{3} & =\left(\begin{array}{cc}
J_{0}\left(k_{2} b_{1}\right) & Y_{0}\left(k_{2} b_{1}\right) \\
\frac{\varepsilon_{2}}{k_{2}} J_{1}\left(k_{2} b_{1}\right) & \frac{\varepsilon_{2}}{k_{2}} Y_{1}\left(k_{2} b_{1}\right)
\end{array}\right) \\
M_{4} & =\left(\begin{array}{cc}
Y_{1}\left(k_{3} b_{1}\right) & -\frac{k_{3}}{\varepsilon_{3}} Y_{0}\left(k_{3} b_{1}\right) \\
-J_{1}\left(k_{3} b_{1}\right) & \frac{k_{3}}{\varepsilon_{3}} J_{0}\left(k_{3} b_{1}\right)
\end{array}\right) .
\end{aligned}
$$

The field distribution and phase velocity of a $\mathrm{TM}_{0 m}$ mode propagating through this dielectric loaded waveguide is determined uniquely by solving the simultaneous Eq. (2). In other words, the propagation characteristics of a $\mathrm{TM}_{0 m}$ mode in this dielectric loaded waveguide are determined by the geometrical parameters $a_{1}, b_{1}$ and $c_{1}$, and the permittivity of a dielectric material $\varepsilon_{2}$. Therefore, unlike conventional all-metal accelerating structures, even if the inner radius of metallic cylinder $c_{1}$ would be arbitrarily selected a value, a desired dispersion relation of the propagating mode can be given by tuning the values of $a_{1}$ and $b_{1}$. The $P_{\text {wall }}$, the wall loss per unit length of this waveguide, can be analytically given by

$$
\begin{aligned}
P_{\text {wall }} & =\frac{R_{s}}{2} \int_{S}|H|^{2} d S \\
& =\frac{\pi}{2} R_{S} c_{1} \frac{\omega^{2} \varepsilon_{3}^{2}}{k_{3}^{2}}\left\{A_{3} J_{1}\left(k_{3} c_{1}\right)+B_{3} Y_{1}\left(k_{3} c_{1}\right)\right\}^{2},
\end{aligned}
$$

where $R_{s}$ is the surface resistance of the metallic cylinder. Equation (3) indicates that the DAA structure is an extremely low rf loss structure as compared with a conventional normal-conducting accelerating structure. Specifically, the wall loss of the higher order $\mathrm{TM}_{0 m}$ mode $(m>1)$ propagating in the dielectric loaded waveguide can be reduced to a value lower than that of the $\mathrm{TM}_{01}$ mode. For example, consider a C-band pillbox cavity made of copper operating in the $\mathrm{TM}_{01}$ mode. Assuming the cavity inner radius to be $R=\rho_{01} c / \omega_{0}$, where $\rho_{01}=2.4048$, the accelerating cell length $L=\lambda_{0} / 2$, and $\mathrm{cw}$ operation at the accelerating gradient $A_{1}=1.0 \mathrm{MV} / \mathrm{m}$, the wall loss on the conducting cylinder can be analytically calculated as $P_{\text {wall }}=1169 \mathrm{~W} / \mathrm{m}$. Here, the $\omega_{0}, c$ and $\lambda_{0}$ represent the angular frequency, the speed of light in vacuum, and the free-space wavelength, respectively, and $\rho_{01}$ is the first root of a Bessel function. On the other hand, in the case of the dielectric loaded waveguide shown in Fig. 1(b), with $a_{1}=0.42 \lambda_{0}, \quad b_{1} / a_{1}=1.2, \quad c_{1} / a_{1}=1.84, \quad \varepsilon_{2} / \varepsilon_{0}=9.6$ where $\varepsilon_{0}$ is permittivity of free space, and operated with the same accelerating gradient $A_{1}=1.0 \mathrm{MV} / \mathrm{m}$, the wall loss of its $\mathrm{TM}_{02}$ mode can be analytically calculated as $P_{\text {wall }}=190 \mathrm{~W} / \mathrm{m}$. This is equivalent to approximately one-sixth of the wall loss in a C-band pillbox cavity. The dielectric loss in this structure can be expressed as

$$
P_{\mathrm{die}}=\frac{1}{2} \omega \varepsilon_{2} \tan \delta \int_{\theta} d \theta \int_{z} d z \int_{a_{1}}^{b_{1}}|\vec{E}|^{2} r d r
$$

where $\tan \delta$ represents the loss tangent of the dielectric material. Considering a total rf loss including the dielectric loss in the dielectric cylinder, Eqs. (3) and (4) result in the conclusion that the total rf loss in this structure is found to be lower than that of a C-band pillbox cavity, if the loss tangent of the dielectric material $\tan \delta$ is $1.0 \times 10^{-4}$ or lower. Recently, ultralow-loss ceramics are actively developed for a wide range of applications, for instance, in wireless communications, and an ultralow-loss alumina having a loss tangent of $7.5 \times 10^{-6}$ has become available [26]. Therefore, it may be possible to build an extremely low-loss accelerating structure using such low-loss ceramics. However, since the phase velocity of the propagating mode in vacuum regions shown in Fig. 1(b) is always greater than the speed of light in vacuum, a slow wave structure is required to use this mode for beam acceleration. To overcome this problem, we propose that ultralow-loss dielectric disks with iris are periodically arranged as a slow wave structure, and a standing wave excited in this structure is used for beam acceleration. The details of the design of such a DAA accelerating cavity are described in the following sections.

\section{REGULAR CELL DESIGN}

The design of the regular cell plays a dominant role in determining the accelerator parameters such as the quality factor and the shunt impedance. Figure 2 shows the axially symmetric cross section of regular cells about the beam axis $z$. The regular cell consists of ultralow-loss dielectric

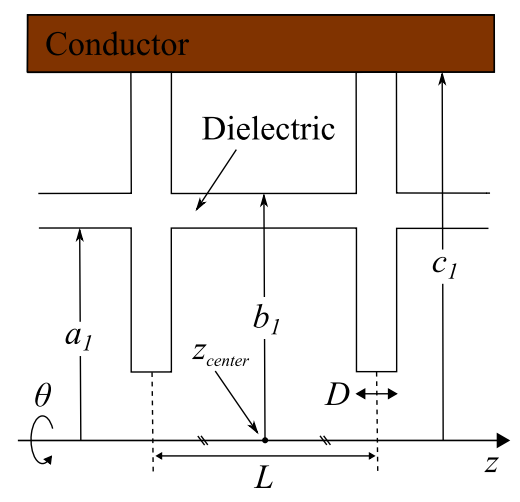

FIG. 2. Longitudinal cross section of the regular cell. 
cylinders with the inner radius $a_{1}$, the outer radius $b_{1}$ and ultralow-loss dielectric disks with a thickness $D$ and beam hole arranged in parallel at a constant interval $L$, in a cylindrical metallic waveguide of the radius $c_{1}$. As discussed in Sec. II, if $c_{1}$ is arbitrarily selected, a desired dispersion relation of the propagating mode can be realized by tuning the value of $a_{1}$ and $b_{1}$. By taking advantage of this fact, in this section, we search for an optimum combination of these geometrical parameters which maximize the quality factor and the shunt impedance of the regular cell part operating at C-band $(5.712 \mathrm{GHz})$ frequency. The accelerator parameters of the regular cell were calculated by using SUPERFISH code.

Here we consider the regular cell of a C-band $(5.712 \mathrm{GHz}) \pi$-mode accelerating structure. With the choice of the $\pi$-mode, the cell length $L$ is given as $L=\lambda_{0} / 2$, where $\lambda_{0}$ is the free-space wavelength. For practical design considerations, we assume to use $\mathrm{TiO}_{2}$-doped $\mathrm{Al}_{2} \mathrm{O}_{3}$ ceramics which has the relative permittivity $\varepsilon_{r}$ of 10.15 and $\tan \delta$ of $7.516 \times 10^{-6}$ [26], and we choose the ceramics disk thickness $D$ as being a quarter $(1 / 4)$ of the resonant wavelength in this dielectric material. The discussion in the previous section has shown that the resonant frequency $f_{0}$ is determined from a combination of $a_{1}, b_{1}$, and $c_{1}$. In particular, the resonant frequency $f_{0}$ is sensitive to $a_{1}$ and $b_{1}$. For that reason, in our design procedure we first fix $a_{1}$ and $c_{1}$, and calculate the value of $b_{1}$ which gives the desired $f_{0}$. Then we examine the quality factor and the shunt impedance expected for a regular cell with the given $a_{1}$ and $c_{1}$. In this calculation, we assumed that the regular cells were infinitely arranged along the beam axis $z$, and the dielectric loss and the wall loss on the metallic cylinder were estimated as if dissipation in the regular cell.

Figure 3 shows the relation between the geometrical parameters $a_{1}, c_{1}$, and the unloaded quality factor, and the shunt impedance of regular cells. In these figures, the white area indicates the parameter combinations where an accelerating mode $\left(\mathrm{TM}_{02}\right.$ mode) that matches the operation frequency could not be found. These simulation results show that there is a possibility to realize a DAA structure with the unloaded quality factor of $Q_{0}=136,000$ and shunt impedance of $R_{\mathrm{sh}}=797 \mathrm{M} \Omega / \mathrm{m}$ by using existing alumina ceramics. They also show that an optimum model can be built without difficulty of fabrication, because the thickness of the dielectric cylinder $\left(b_{1}-a_{1}\right)$ is about $0.07 \lambda_{0}$ and is thick enough for machining with good precision.

Figures 4 and 5 visualize the $\mathrm{TM}_{02}-\pi$ mode standing wave for beam acceleration that is excited in this regular cell. The contour plots of the electric field distribution $E_{z}(r, \theta, z)$ and the magnetic field distribution $H_{\theta}(r, \theta, z)$ of the $\pi$ mode in the regular cell are shown in Fig. 4. These figures are the results of a three-dimensional simulation performed with CST MicROWAVE STUDIO on the cell model which has a highest quality factor. Figure 5 shows the normalized longitudinal electric field and rotating magnetic
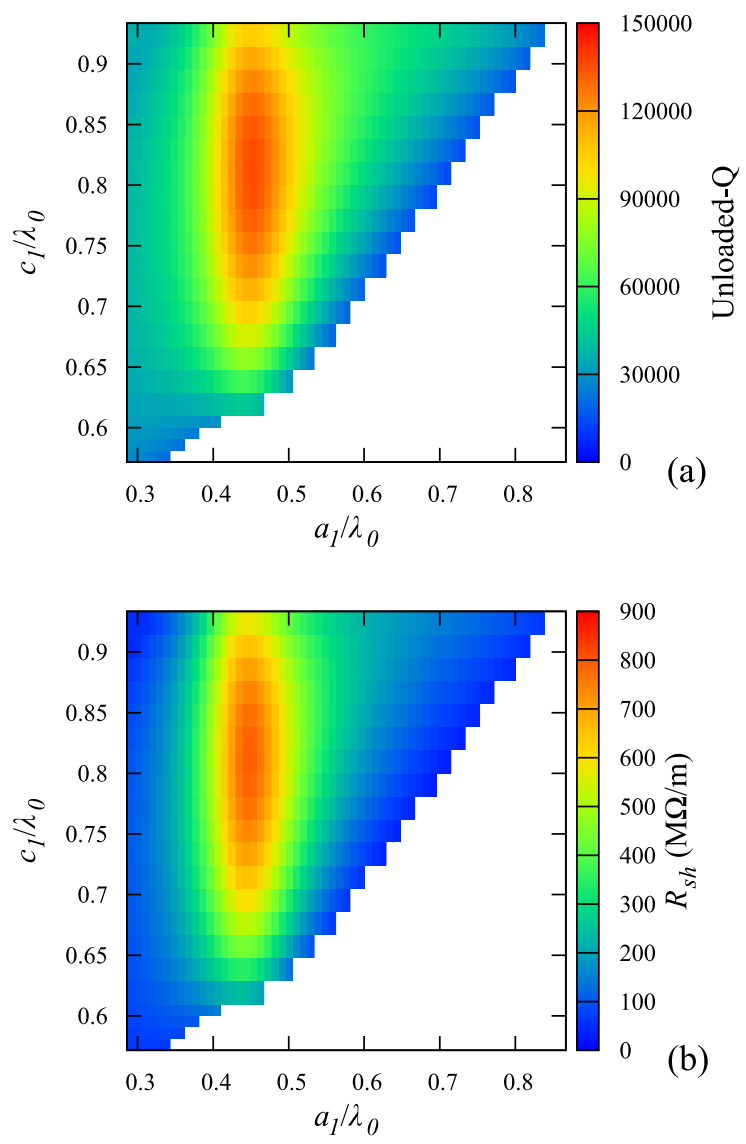

FIG. 3. (a) The dependence of unloaded quality factor of the DAA type regular cell on the geometrical parameters, $a_{1}$ and $c_{1}$. (b) The dependence of shunt impedance of the DAA type regular cell on the geometrical parameters, $a_{1}$ and $c_{1}$.
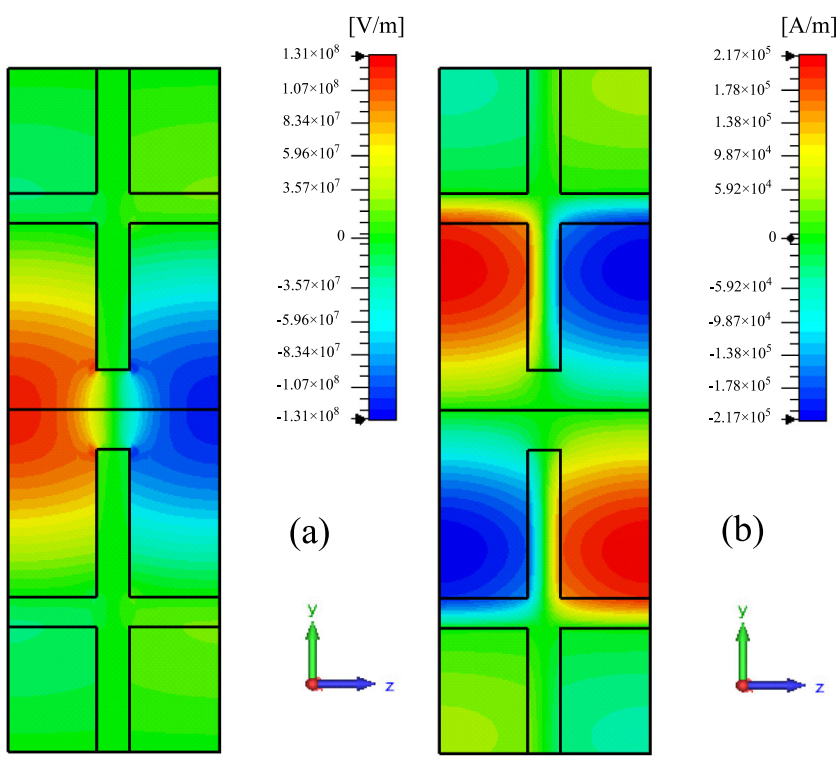

FIG. 4. Contour plots of the field distribution of the accelerating mode in the regular cell. (a) Electric field distribution $E_{z}(r, \theta, z)$. (b) Magnetic field distribution $H_{\theta}(r, \theta, z)$. These plots of the field intensity are shown in arbitrary units. 


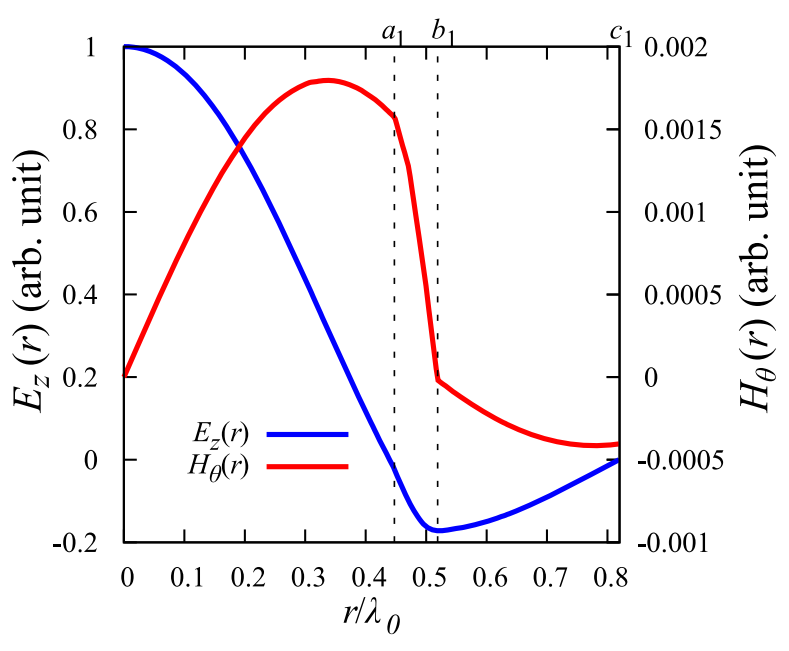

FIG. 5. Normalized longitudinal electric field, $E_{z}\left(r, z_{\text {center }}\right)$ (blue line) and rotating magnetic field, $H_{\theta}\left(r, z_{\text {center }}\right)$ (red line) of the accelerating mode along the $r$ axis.

field along the $r$ axis at the center position of the regular cell on $z$ axis $z_{\text {center }}$.

With the $\left|H_{\theta \text {,max }}\left(r, \theta, z_{\text {center }}\right)\right|$ being the maximum value of the rotating magnetic field within $0<r<c_{1}$ at $z_{\text {center }}$, the ratio of the rotating magnetic field amplitude on the $c_{1}$ surface $\left|H_{\theta}\left(c_{1}, \theta, z_{\text {center }}\right)\right| /\left|H_{\theta, \max }\left(r, \theta, z_{\text {center }}\right)\right|$ is 0.225 in this regular cell. This ratio is much lower than the case for a simple $\mathrm{TM}_{01} \mathrm{C}$-band pillbox cavity which gives approximately 0.9 . It implies that the surface current on the conducting cylinder of a DAA structure can be reduced much lower than that of a simple $\mathrm{TM}_{01} \mathrm{C}$-band pillbox cavity.

Equation (3) shows that the wall loss on the conducting cylinder is scaled in proportion to the cavity radius $c_{1}$ and the square of $\left|H_{\theta}\left(c_{1}, \theta, z_{\text {center }}\right)\right|$. Therefore, while the $c_{1}$ of the DAA structure is about twice as large as the cavity radius of a simple $\mathrm{TM}_{01} \mathrm{C}$-band pillbox cavity, the square of the ratio $\left|H_{\theta}\left(c_{1}, \theta, z_{\text {center }}\right)\right| /\left|H_{\theta, \max }\left(r, \theta, z_{\text {center }}\right)\right|$ is so low that the wall loss on the conducting cylinder of DAA structure can be much reduced. The slow wave structure of the DAA structure consists of the periodically arranged ultralow-loss ceramic disks. This leads to an additional reduction of the $\mathrm{rf}$ loss in each disk structures as compared to that of metallic disks in the conventional allmetal accelerating structures. For example, the result of the SUPERFISH simulation indicates that the total dielectric loss in the optimized regular cell is about a half of the wall loss on the conducting cylinder of its own cell.

Figure 6 shows the distributions of electric and magnetic energy density of $\mathrm{TM}_{02}$ mode in the regular cell. These simulation results indicates that most of the rf energy is stored in the vacuum region $(i=1)$ and can be used effectively for beam acceleration.

In summary, the total $\mathrm{rf}$ loss including both the wall loss on the conducting cylinder and dielectric loss in the DAA structure can be drastically reduced, so that the

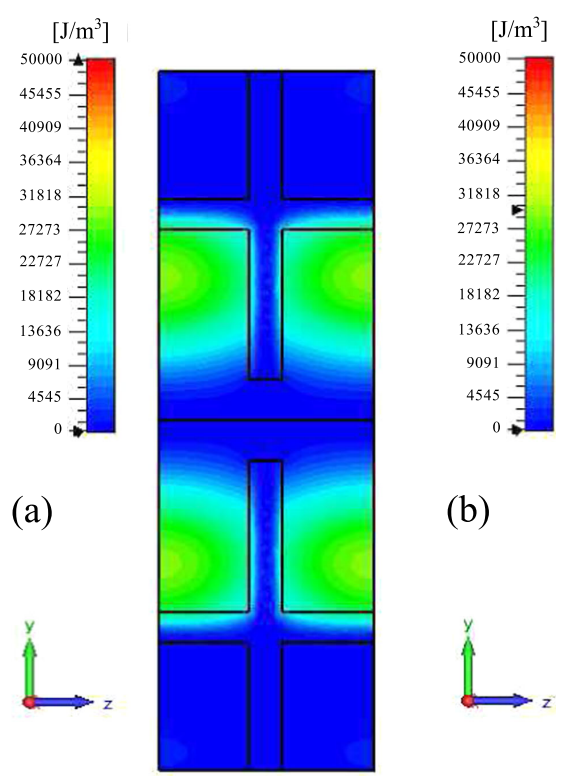

FIG. 6. (a) Energy density in an electric field of the accelerating mode. (b) Energy density in a magnetic field of the accelerating mode. These distributions of energy density are shown in arbitrary units.

DAA structure has both an extremely high quality factor and a very high shunt impedance at room temperature.

\section{END CELL DESIGN}

The end cells, arranged at both ends of the DAA cavity, are provided to reduce the $\mathrm{rf}$ dissipation on the surface of both conducting end plates. Figure 7 shows an upper half part of the longitudinal cross section of an end cell. The end cell consists of a multilayered cylindrical structure and a disk with iris which is made of an ultralow-loss dielectric material, the same as what is used for regular cells. By selecting the geometrical parameters, the surface magnetic field in the $\theta$ direction on the conducting end plates can be made lower than the case without the end cell structures. This helps to maintain the desired feature of the low-loss and high quality factor for the DAA as a complete

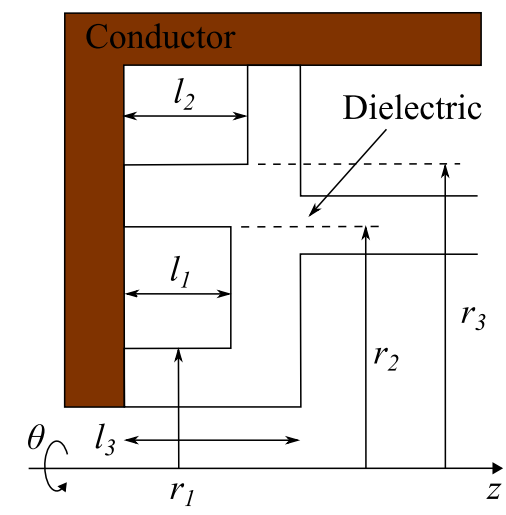

FIG. 7. Longitudinal cross section of the end cell. 


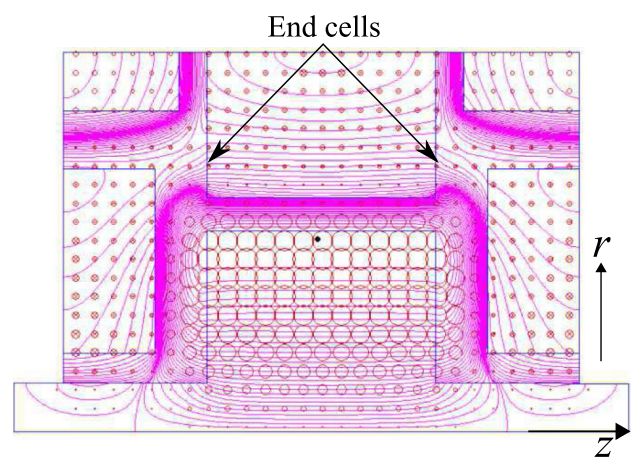

FIG. 8. Field distribution of an optimized single cell DAA cavity. Red lines indicate the electric lines of force. The circle symbols indicate the axisymmetric magnetic field of accelerating mode and the sizes of the circles represent the magnetic field strength.

accelerating structure. In this section, we discuss on a calculation with SUPERFISH of a single cell DAA cavity which has a regular cell and two end cell structures, and demonstrate the benefit of the end cell structure. More specifically, six geometrical parameters shown in Fig. 7 are optimized so that the wall loss on the conducting end plates can be minimized by using the down-hill simplex method.

Figure 8 shows a SUPERFISH simulation result for the accelerating mode of an optimized single cell DAA cavity. These red lines indicate the electric lines of force. The circle symbols indicate the axisymmetric magnetic field of accelerating mode and the sizes of the circles represent the magnetic field strength. This simulation result shows that most of the rf energy is stored near the beam axis in a part of the regular cell and the stored energy in vacuum regions between the conducting end plate and the dielectric disk is much lower. Consequently, the total wall loss in the optimized single cell cavity can be about $60 \%$ lower than that of a single cavity model which is electrically shorted by mere conducting end plates without some dielectric structures as end cell structures.

The accelerator parameters corresponding to the calculations represented in the Fig. 8 structure are given in Table I.

TABLE I. The parameters of the accelerating structure that is illustrated in Fig. 8.

\begin{tabular}{lc}
\hline \hline Parameter & Single cell cavity \\
\hline Dielectric Material & $\mathrm{TiO}_{2}$-doped alumin \\
$\varepsilon_{r}[26]$ & 10.15 \\
$\tan \delta[26]$ & $7.516 \times 10^{-6}$ \\
Operation frequency & $5.712 \mathrm{GHz}$ \\
Operating temperature & $20^{\circ} \mathrm{C}$ \\
$Q_{0}$ & 81956 \\
$R_{\mathrm{sh}}$ & $377 \mathrm{M} \Omega / \mathrm{m}$ \\
\hline \hline
\end{tabular}

\section{PROTOTYPE DESIGN OF DAA STRUCTURE}

In this section, a prototype DAA design which adopts the ideas of "regular" and "end" cells as discussed in the previous two sections is described in detail. Due to the availability of C-band klystrons operating at a pulse duration over $10 \mu \mathrm{s}$ at KEK, we focused our cavity design for an operating frequency of $5.712 \mathrm{GHz}$ so that the modeled cavity can be high-power tested. Relative permittivity and loss tangent of the dielectric regions were set at measured values of $\varepsilon_{r}=$ 10.15 and $\tan \delta=7.516 \times 10^{-6}$ [26]. As an example, we present a design of a " 5 cell" DAA structure in the following. The geometrical parameters were optimized in accordance with discussion in Sec. III so that the unloaded quality factor and shunt impedance might become maximized by using the down-hill simplex method. All of the accelerator parameters were calculated by the SUPERFISH code.

Table II presents the basic cavity and accelerator parameters of an optimized 5 cell DAA structure. An extremely high quality factor and a very high shunt impedance are shown to be expected. The quality factor of 122,000 is almost 10 times higher than that of the conventional diskloaded copper structures. Moreover, the shunt impedance of $656 \mathrm{R} \Omega / \mathrm{m}$ is also several times higher than that of the conventional C-band accelerating structure. These values correspond to the highest level of cavity performance operable at room temperature, and they imply that high accelerating gradient can be provided with much less if power in the DAA structure.

Figure 9 shows the electric field lines and the normalized longitudinal electric field strength of the accelerating mode excited in the 5 cell DAA structure. The magnetic symmetry planes are located on the middle plane of each dielectric disk, and the accelerating mode $\left(\mathrm{TM}_{02}-\pi\right.$ mode $)$ is excited in each regular cell.

The accelerator parameters of the prototype model shown in Table II were calculated by using the values of relative permittivity and the loss tangent of $\mathrm{TiO}_{2}$-doped $\mathrm{Al}_{2} \mathrm{O}_{3}$ ceramics in [26]. Figure 10 shows how the varying $\tan \delta$ influences the unloaded quality factor and shunt

TABLE II. Basic cavity and accelerator parameters of an optimized 5 cell DAA structure.

\begin{tabular}{lc}
\hline \hline Parameter & 5 cell DAA structure \\
\hline Dielectric material & $\mathrm{TiO}_{2}$-doped alumin \\
$\varepsilon_{r}[26]$ & 10.15 \\
$\tan \delta[26]$ & $7.516 \times 10^{-6}$ \\
Accelerator type & $\mathrm{Standing}^{-6}$ wave type \\
Accelerating mode & $\mathrm{TM}_{02}-\pi$ mode \\
Operation frequency & $5.712 \mathrm{GHz}$ \\
Total cavity length & $157.5 \mathrm{~mm}$ \\
Number of accelerating cells & 5 \\
Operating temperature & $20^{\circ} \mathrm{C}$ \\
$Q_{0}$ & 122,000 \\
$R_{\mathrm{sh}}$ & $656 \mathrm{M} \Omega / \mathrm{m}$ \\
\hline \hline
\end{tabular}




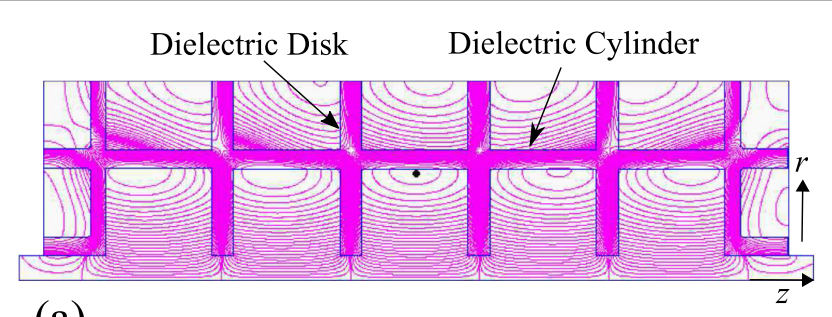

(a)

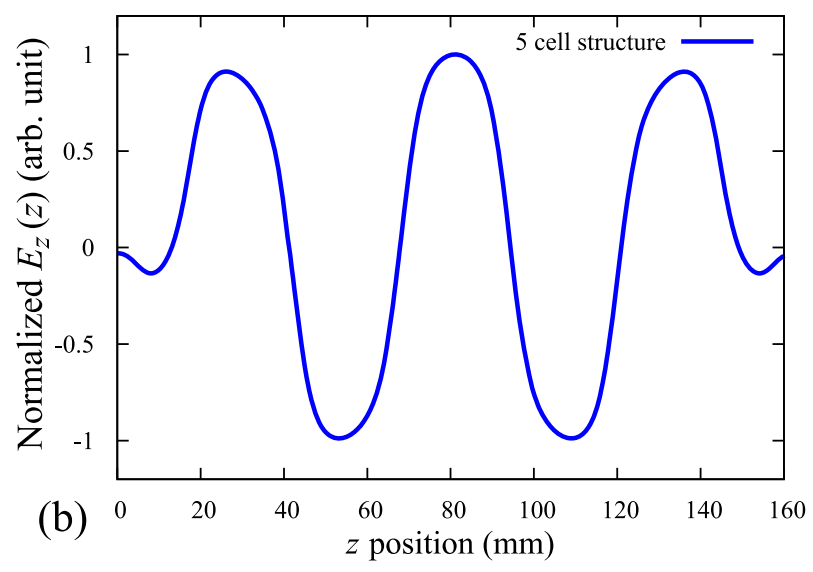

FIG. 9. (a) Electric field lines of the accelerating mode excited in the 5 cell DAA structure. (b) Normalized longitudinal electric field strength in the 5 cell DAA structure.

impedance of the same DAA cavity, respectively. In these figures, the horizontal axis indicates the loss tangent $\tan \delta$ of a dielectric material and the vertical axes indicate unloaded quality factor and shunt impedance of the 5 cell prototype model, respectively. In order to illustrate the benefit of accelerator parameters in the DAA structures, the unloaded $Q$ (blue dashed line) and shunt impedance (red dashed line) of conventional C-band copper cavity with the cavity inner radius $R=0.39 \lambda_{0}$, the iris radius $h=0.095 \lambda_{0}$ and the

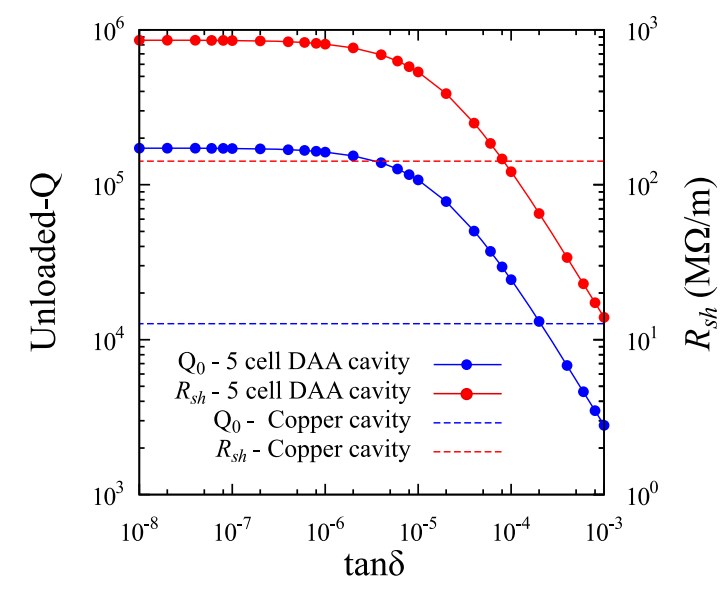

FIG. 10. Unloaded quality factor and shunt impedance of the 5 cell DAA cavity as a function of loss tangent of a dielectric material. accelerating cell length $L=\lambda_{0} / 2$ are also shown in Fig. 10. Basically, as the loss tangent is decreased, both the unloaded quality factor and the shunt impedance are increased. As shown in Fig. 10, they become saturated for the loss tangent values below $10^{-6}$. It means that dielectric loss becomes negligibly small as compared with the conducting wall loss and these accelerator parameters of the DAA structure are limited by the electrical properties of the metallic enclosure. This shows that it is desirable to develop new ultralow-loss dielectric materials which have a loss tangent of about $1.0 \times 10^{-6}$ at room temperature. Assuming that the loss tangent is zero, in other words, dielectric loss is zero in the DAA cavity, unloaded quality factor and shunt impedance increased to $Q_{0}=178,000$ and $R_{\mathrm{sh}}=906 \mathrm{M} \Omega / \mathrm{m}$, respectively.

\section{CONCLUSIONS}

In this paper, the dielectric assist accelerating (DAA) structure has been proposed as a new type of DLA structure to improve both its quality factor and shunt impedance. This new structure is a higher-order $\mathrm{TM}_{02 n}$ mode standing-wave accelerating structure and consists of ultralow-loss dielectric cells periodically arranged in a metallic enclosure. In analytical calculations, we have shown that the wall loss of $\mathrm{TM}_{02}$ propagation mode in this structure is extremely reduced, since the surface current on the conducting cylinder can be reduced much lower than that of conventional normal-conducting cavities. In addition, with the computer simulations we have shown that the 5 cell DAA structure of Fig. 9 will have the $Q_{0}$ value of about 10 times or higher and the $R_{\mathrm{sh}}$ value of several times higher than those of a conventional C-band accelerating structure. These results indicate that an accelerating gradient can be realized with much less rf power and at reduced operation cost. Moreover, the DAA structure can be fabricated at relatively low cost, since it has a simple cell structure composed of a ceramic material. In addition, it can be operated at room temperature, and therefore, it is expected to be applicable to both high energy accelerators and industrial applications such as medical linacs [5,6] and others.

According to the preceding studies of DLA structures, a dielectric joint breakdown occurred at microgaps [27] and rf power absorption caused by single-surface multipactor was experimentally found $[28,29]$. Therefore, in order to realize a high-gradient DAA structure, it is essential to develop a careful design of input coupler and dielectric cell assembly to avoid the rf breakdown and to mitigate multipactoring.

Despite these remaining issues, on the basis of the merits of DAA structures as we have discussed in previous sections, the DAA structures have potential real-life applications. For instance, it may be deployed in a compact and highly efficient accelerator for low-energy beam applications. In the case of the 5 cell DAA structure of Fig. 9, the average accelerating gradient of over $1 \mathrm{MV} / \mathrm{m}$ can be 
achieved with a rf power less than $1 \mathrm{~kW}$. Thus, with a DAA it may become possible to build a portable $\mathrm{x}$-ray source much more compact than those with conventional systems [30,31].

In order to realize the DAA structure having the higher quality factor and shunt impedance, the development of ultralow-loss ceramics is highly desirable. The simulation results of the relationship between accelerator parameters of the 5 cells DAA cavity and loss tangent of dielectric material predict that dielectric loss becomes negligibly small as compared with the conducting wall loss, if ultralow-loss ceramic with loss tangent below $10^{-6}$ could be used for these cavity cells. Therefore, development of new ceramics or high quality ceramics of which loss tangent is lower than $10^{-6}$ at room temperature would be a significant benefit for the DAA structure.

\section{ACKNOWLEDGMENTS}

The authors would like to thank T. Natsui and N. Toge for helpful advice. This work has been supported by Grantin-Aid for JSPS Fellows Grant No. 13J08681.

[1] S. Dobert, A. Grudiev, G. Riddone, W. Wuensch, R. Zennaro, T. Higo, S. Matsumoto, K. Yokoyama, C. Adolphsen, F. Wang, and J. Wang, First high power tests of clic prototype accelerating structures with hom waveguide damping, in Proceedings of the 25th International Linear Accelerator Conference, LINAC-2010, Tsukuba, Japan (KEK, Tsukuba, Japan, 2010), pp. 208-210.

[2] T. Higo, Progress of x-band accelerating structures, in Proceedings of the 25th International Linear Accelerator Conference, LINAC-2010, Tsukuba, Japan (Ref. [1]), pp. 1038-1042.

[3] R. C. Sethi, Electron beam accelerators for materials processing: A barc scenatrio, in Proceedings of the APAC 2004, Gyeongju, Korea (Pohang Accelerator Laboratory, Gyeongju, 2004), pp. 708-710.

[4] L. Auditore, R. C. Barna, D. DePasquale, A. Italiano, A. Trifiro, and M. Trimarchi, Pulsed $5 \mathrm{MeV}$ standing wave electron linac for radiation processing, Phys. Rev. ST Accel. Beams 7, 030101 (2004).

[5] E. Tanabe, Y. Fineberg, H. Matsumoto, and T. Shintake, Medical applications of C-band accelerator technologies, in Proceedings of the 1998 Linear Accelerator Conference, Argonne, IL, USA (NTIS, Springfield, 2004), pp. 627-629.

[6] J. Hyun, M. Yoshida, M. Satoh, T. Sakai, Y. Hayakawa, T. Tanaka, K. Hayakawa, I. Sato, and K. Endo, Design of wavelength tunable coherent $\mathrm{x}$-ray source, in Proceedings of IPAC2015, Richmond, VA, USA (IEEE, Piscataway, NJ, 2015), pp. 1604-1606.

[7] W. Gai, R. Konecny, and J. Simpson, Externally powered dielectric loaded waveguides as accelerating structures, in Proceedings of the 1997 Particle Accelerator Conference, Vancouver, Canada (IEEE, Piscataway, NJ, 1998), pp. 636-638.
[8] P. Zou, W. Gai, R. Konecny, X. Sun, T. Wong, and A. Kanareykin, Externally powered dielectric loaded waveguides as accelerating structures, Rev. Sci. Instrum. 71, 2301 (2000).

[9] W. Liu, C. Jing, W. Gai, R. Konecny, and J. G. Power, New rf design for $11.4 \mathrm{GHz}$ dielectric loaded accelerator, in Proceedings of the 2003 Particle Accelerator Conference, Portland, Oregon, USA (IEEE, Piscataway, NJ, 2003), pp. 1810-1812.

[10] S. Frankel, Tm 0,1 mode in circular wave guides with two coaxial dielectrics, J. Appl. Phys. 18, 650 (1947).

[11] G. G. Bruck and E. R. Wicher, Slow transverse magnetic waves in cylindrical guides, J. Appl. Phys. 18, 766 (1947).

[12] A. A. Oliner, Remarks on slow waves in cylindrical guides, J. Appl. Phys. 19, 109 (1948).

[13] T.-B. Zhang, J. L. Hirshfield, T. C. Marshall, and B. Hafizi, Stimulated dielectric wake-field accelerator, Phys. Rev. E 56, 4647 (1997).

[14] K. DeckerFrench, Development of a dielectric loaded rf cavity for a muon accelerator, Nucl. Instrum. Methods Phys. Res., Sect. A 624, 731 (2010).

[15] C. Jing, A. Kanareykin, S. Kazakov, W. Liu, E. Nenasheva, P. Schoessow, and W. Gai, Development of a dual-layered dielectric-loaded accelerating structure, Nucl. Instrum. Methods Phys. Res., Sect. A 594, 132 (2008).

[16] P. Zou, L. Xiao, X. Sun, and W. Gai, A hybrid dielectric and iris loaded periodic accelerating structure, in Proceedings of the 2001 Particle Accelerator Conference, Chicago (IEEE, Piscataway, NJ, 2001), pp. 3966-3968.

[17] C. Jing, W. Liu, W. Gai, J. Power, and T. Wong, Mode analysis of a multilayered dielectric-loaded accelerating structure, Nucl. Instrum. Methods Phys. Res., Sect. A 539, 445 (2005).

[18] C. Jing, W. Liu, W. Gai, J. G. Power, and A. Kanareykin, $34.272 \mathrm{GHz}$ multilayered dielectric-loaded accelerating structure, in Proceedings of the 2005 Particle Accelerator Conference, Knoxville, Tennessee (IEEE, Piscataway, NJ, 2005), pp. 1592-1594.

[19] E. S. Kim and C. J. Jeon, Microwave dielectric properties of atio3 ( $a=$ ni, mg, co, mn) ceramics, J. Eur. Ceram. Soc. 30, 341 (2010).

[20] T. Tsunookaa, M. Androua, Y. Higashidaa, H. Sugiurab, and $\mathrm{H}$. Ohsatoc, Effects of $\mathrm{TiO}_{2}$ on sinterability and dielectric properties of high-q forsterite ceramics, J. Eur. Ceram. Soc. 23, 2573 (2003).

[21] H. Ohsato, T. Tsunooka, T. Sugiyama, K. Kakimoto, and H. Ogawa, Forsterite ceramics for millimeterwave dielectrics, J. Electroceram. 17, 445 (2006).

[22] N. M. Alford and S. J. Penn, Sintered alumina with low dielectric loss, J. Appl. Phys. 80, 5895 (1996).

[23] C. L. Huang, J. J. Wang, and C. Y. Huang, Sintering behavior and microwave dielectric properties of nano alpha-alumina, Mater. Lett. 59, 3746 (2005).

[24] C. L. Huang, J. J. Wang, and C. Y. Huang, Microwave dielectric properties of sintered alumina using nano-scaled powders of a alumina and $\mathrm{TiO}_{2}$, J. Am. Ceram. Soc. 90, 1487 (2007).

[25] E. Chojnacki, W. Gai, C. Ho, R. Konecny, S. Mtingwa, J. Norem, M. Rosing, P. Schoessow, and J. Simpson, 
Measurement of deflection-mode damping in an accelerating structure, J. Appl. Phys. 69, 6257 (1991).

[26] J. D. Breeze, X. Aupi, and N. M. Alford, Ultralow loss polycrystalline alumina, Appl. Phys. Lett. 81, 5021 (2002).

[27] C. Jing, A. Kanareykin, R. Konecny, J. G. Power, S. H. Gold, and S. Kazakov, Progress towards a gap free dielectric-loaded accelerator, in Proceedings of the 2007 Particle Accelerator Conference, Albuquerque, New Mexico, USA (IEEE, Piscataway, NJ, 2007), pp. 3151-3153.

[28] J. G. Power, W. Gai, S. H. Gold, A. K. Kinkead, R. Konecny, C. Jing, W. Liu, and Z. Yusof, Observation of multipactor in an alumina-based dielectric-loaded accelerating structure, Phys. Rev. Lett. 92, 164801 (2004).

[29] C. Jing, J. G. Power, R. Konecny, W. Gai, S. H. Gold, and A.K. Kinkead, Progress on high power tests of dielectric-loaded accelerating structures, in Proceedings of the 2005 Particle Accelerator Conference, Knoxville, Tennessee (IEEE, Piscataway, NJ, 2007), pp. 1566-1568.

[30] T. Yamamoto, T. Natsui, F. Sakamoto, M. Uesaka, N. Nakamura, and E. Tanabe, Development of portable $\mathrm{x}$-band linac $\mathrm{x}$-ray source for non-destructive testing, in Proceedings of the Joint International Workshop: Nuclear Technology and Society-Needs for Next Generation, Berkeley, California (Berkeley, California, 2008).

[31] S. Xiang, T. Dechun, J. Qingxiu, L. Yuzheng, S. Jingqing, H. Shaoguang, D. Taibin, D. Xiuming, C. Bingyi, L. Yuezeng, Z. Yang, and H. Wensheng, A portable x-band on-axis standing wave linac structure, in Proceedings of the 1997 Particle Accelerator Conference, Vancouver, Canada (Ref. [7]), pp. 1221-1223. 